\title{
Dendrites and measures with discrete spectrum
}

\author{
MAGDALENA FORYŚ-KRAWIEC $†$, JANA HANTÁKOVÁ †, JIŘÍ KUPKA§, \\ PIOTR OPROCHA(D $\dagger \S$ and SAMUEL ROTH(D† \\ $\dagger$ Faculty of Applied Mathematics, AGH University of Science and Technology, \\ al. Mickiewicza 30, 30-059 Kraków, Poland \\ (e-mail:maforys@agh.edu.pl,oprocha@agh.edu.pl) \\ $\$$ Mathematical Institute Silesian University in Opava, Na Rybníčku 1, \\ 74601 Opava, Czech Republic \\ (e-mail: jana.hantakova@math.slu.cz, samuel.roth@math.slu.cz) \\ $\S$ Centre of Excellence IT4Innovations - Institute for Research and Applications of Fuzzy \\ Modeling, University of Ostrava, 30. dubna 22, 70103 Ostrava 1, Czech Republic \\ (e-mail: jiri.kupka@osu.cz)
}

(Received 4 April 2021 and accepted in revised form 26 October 2021)

Abstract. We are interested in dendrites for which all invariant measures of zero-entropy mappings have discrete spectrum, and we prove that this holds when the closure of the endpoint set of the dendrites is countable. This solves an open question which has been around for awhile, and almost completes the characterization of dendrites with this property.

Key words: dendrite, discrete spectrum, topological entropy, minimal set 2020 Mathematics Subject Classification: 37B40, 37B45, 54F50

\section{Introduction}

A dynamical system is a pair $(X, f)$, where $X$ is a compact metric space and $f: X \rightarrow X$ is a continuous map. A continuum is a compact connected metric space. Throughout this paper, we assume $X$ is a dendrite, that is, a locally connected continuum containing no simple closed curve.

The main motivation of the paper can be derived from the Möbius disjointness conjecture proposed by Sarnak in 2009 [23]. By topological arguments, the conjecture was confirmed on various one-dimensional spaces: the interval [14], the circle [8], topological graphs [16], some dendrites [11], etc. However, using ergodic theory, it was proved that if all invariant measures have discrete spectrum, then the conjecture also holds (see e.g. [13, Theorem 1.2]). This leads to a natural question, what can be said about the spectrum of measures for zero-entropy maps in the above-mentioned spaces. In [17], the authors 
confirmed that, indeed, maps on topological graphs with zero entropy can have only invariant measures with discrete spectrum. This motivated the following open question [17, Question 1.1].

Question 1.1. Which one-dimensional continua $X$ have the property that every invariant measure of $(X, f)$ has discrete spectrum, assuming $f$ is a zero-entropy map?

Similar questions, however, were stated even before, for example in [24] from 1982, where the author asked whether every ergodic invariant measure in a mean equicontinuous system has discrete spectrum. The authors of [17] partially answered this question by showing that the result holds for zero-entropy maps on quasi-graphs $X$, and it was completely answered in the affirmative in 2015 in [18]. Let us mention at this point that continua satisfying the condition in Question 1.1 cannot be too complex. It was shown in [17] that if a dendrite has an uncountable set of endpoints, then it supports a plethora of maps with zero topological entropy possessing invariant measures which do not have discrete spectrum. Then in the realm of dendrites, only those with a countable set of endpoints can be examples in Question 1.1.

In this paper, we study the dynamics of zero-entropy maps on dendrites for which the endpoint set has a countable closure. In $\S 3$, we build on results from [3, 4] and show that every recurrent point is in fact minimal (Theorem 3.6), which generalizes a well-known property of zero-entropy interval maps. In $\S 4$, we use this result together with a characterization of minimal $\omega$-limit sets from [4] to show that every invariant measure has discrete spectrum (Theorem 4.3) in the case of these dendrites. Our results almost completely characterize dendrites for which all invariant measures of zero-entropy mappings have discrete spectrum. We leave unsolved the case of dendrites for which the endpoint set is countable but has an uncountable closure. We strongly believe that in the case of these dendrites, the analog of Theorem 3.6 also holds, because all known examples seem to confirm that. Unfortunately, we were not able to find a good argument to justify this statement. Structural properties of (other) one-dimensional continua that may serve as positive examples in Question 1.1 are yet to be understood.

\section{Preliminaries}

Let $(X, f)$ be a dynamical system and $x \in X$. The orbit of $x$, denoted by $\operatorname{Orb}_{f}(x)$, is the set $\left\{f^{n}(x): n \geq 0\right\}$, and the $\omega$-limit set of $x$, denoted by $\omega_{f}(x)$, is defined as the intersection $\bigcap_{n \geq 0} \overline{\left\{f^{m}(x): m \geq n\right\}}$. It is easy to check that $\omega_{f}(x)$ is closed and strongly $f$-invariant, that is, $f\left(\omega_{f}(x)\right)=\omega_{f}(x)$. The point $x$ is periodic $(x \in \operatorname{Per}(f))$ if $f^{p}(x)=x$ for some $p \in \mathbb{N}$, where the smallest such $p$ is called the period of $x$. Note that throughout this paper, $\mathbb{N}$ denotes the set of positive integers. The point $x$ is recurrent $(x \in \operatorname{Rec}(f))$ if $x \in \omega_{f}(x)$. The orbit of a set $A \subset X$, denoted $\operatorname{Orb}_{f}(A)$, is the set $\bigcup_{n \geq 0} f^{n}(A)$, and $A$ is called invariant if $f(A) \subseteq A$. A set $M$ is minimal if it is non-empty, closed, invariant, and does not have a proper subset with these three properties. It can be equivalently characterized by $M=\omega_{f}(x)$ for every $x \in M$. A point is minimal if it belongs to a minimal set. 
Recall that a dendrite is a locally connected continuum $X$ containing no homeomorphic copy of a circle. A continuous map from a dendrite into itself is called a dendrite map. For any point $x \in X$, the $\operatorname{order}$ of $x$, denoted by ord $(x)$, is the number of connected components of $X \backslash\{x\}$. Points of order 1 are called endpoints while points of order at least 3 are called branch points. By $E(X)$ and $B(X)$ we denote the set of endpoints and branch points, respectively. In this paper, we especially focus on dendrites in which $E(X)$ has countable closure. These dendrites are a special case of a tame graph, as introduced in [5]. Note that when $\overline{E(X)}$ is countable, so also is $\overline{B(X) \cup E(X)}$, because $B(X)$ is countable in any dendrite and has accumulation points only in $\overline{E(X)}$.

For any two distinct points $x, y \in X$, there exists a unique arc $[x, y] \subset X$ joining those points. A free arc is an arc containing no branch points. We say that two arcs $I, J$ form an arc horseshoe for $f$ if $f^{n}(I) \cap f^{m}(J) \supset I \cup J$ for some $n, m \in \mathbb{N}$, where $I, J$ are disjoint except possibly at one endpoint. Denote by $h_{\text {top }}(f)$ the topological entropy of a dendrite map $f$ (for the definition, see [1, 7, 9]). We will frequently use the fact that for dendrite maps, positive topological entropy is implied by the existence of an arc horseshoe [15].

The set of all Borel probability measures over $X$ is denoted by $M(X)$, and $M_{f}(X) \subset$ $M(X)$ denotes the set of all elements of $M(X)$ invariant with respect to the map $f$. The set of all ergodic measures in $M_{f}(X)$ is denoted by $M_{f}^{e}(X)$. We say that a finite measure $\mu$ on $X$ is concentrated on $A \subset X$ if $\mu(A)=\mu(X)$. It is well known that $M(X)$ endowed with the weak-* topology is a compact metric space and that $M_{f}(X)$ is its closed subset. We say that $\mu \in M_{f}(X)$ has discrete spectrum, if the linear span of the eigenfunctions of $U_{f}$ in $L_{\mu}^{2}(X)$ is dense in $L_{\mu}^{2}(X)$, where as usual $U_{f}$ denotes the Koopman operator: $U_{f}(\varphi)=$ $\varphi \circ f$ for every $\varphi \in L_{\mu}^{2}(X)$. We refer the reader to $[10,26]$ for standard monographs on ergodic theory and entropy.

\section{Recurrence and minimality in dendrites with $\overline{E(X)}$ countable}

First we recall the following results by Askri on the structure of minimal $\omega$-limit sets in a special class of dendrite maps.

Proposition 3.1. [4, Proposition 3.4] Let $X$ be a dendrite such that $E(X)$ is countable and let $f: X \rightarrow X$ be a continuous map with zero topological entropy. If $M=\omega_{f}(x)$ is an infinite minimal $\omega$-limit set for some $x \in X$, then for every $k \geq 1$ there is an f-periodic subdendrite $D_{k}$ of $X$ and an integer $n_{k} \geq 2$ with the following properties:

(1) $D_{k}$ has period $\alpha_{k}:=n_{1} n_{2} \ldots n_{k}$;

(2) for $i \neq j \in\left\{0, \ldots, \alpha_{k}-1\right\}, f^{i}\left(D_{k}\right)$ and $f^{j}\left(D_{k}\right)$ are either disjoint or intersect at one common point;

(4) $M \subset \bigcap_{k \geq 0} \operatorname{Orb}_{f}\left(D_{k}\right)$;

(5) $f\left(M_{i}^{k}\right)=M_{i+1 \bmod \alpha_{k}}^{k}$, where $M_{i}^{k}=M \cap f^{i}\left(D_{k}\right)$ for all $k$ and all $0 \leq i \leq \alpha_{k}-1$.

While equation (5) is not directly stated in [4], it is an obvious consequence of the other statements. 
Implicit in Proposition 3.1 is the idea that the minimal set $M$ has an odometer as a factor. Our next lemma shows that when $E(X)$ has countable closure, the factor map is invertible except on a countable set.

Given an increasing sequence $\left(\alpha_{k}\right)$ with $\alpha_{k} \mid \alpha_{k+1}$ for all $k$, we define the group $\Omega=$ $\Omega\left(\alpha_{k}\right)$ of all $\theta \in \prod_{k=0}^{\infty} \mathbb{Z} / \alpha_{k} \mathbb{Z}$, such that $\theta_{k+1}$ is congruent to $\theta_{k}$ modulo $\alpha_{k}$ for all $k$, and we let $\tau$ denote the group rotation $\tau(\theta)=\theta+(1,1,1, \ldots)$. Then $(\Omega, \tau)$ is called the odometer associated to the sequence $\left(\alpha_{k}\right)$.

Lemma 3.2. Let $X, f, M,\left(D_{k}\right),\left(\alpha_{k}\right)$ be as in Proposition 3.1 and suppose that $\overline{E(X)}$ is countable. Then the following hold.

(1) The sets $J_{\theta}=\bigcap_{k} f^{\theta_{k}}\left(D_{k}\right), \theta \in \Omega$, are closed, connected, and pairwise disjoint.

(2) There is a factor map $\pi:(M, f) \rightarrow(\Omega, \tau)$ which takes the value $\theta$ on $M \cap J_{\theta}$.

(3) Each fiber $\pi^{-1}(\theta), \theta \in \Omega$, is countable, and all but countably many of these fibers are singletons.

Proof. It is clear from Proposition 3.1 that each set $J_{\theta}$ is closed, connected, and has non-empty intersection with $M$. It is also clear that $f\left(J_{\theta}\right)=J_{\tau(\theta)}$. However, because the sets $f^{i}\left(D_{k}\right) \cap f^{j}\left(D_{k}\right)$ are allowed to intersect at a point, it is not clear if the sets $J_{\theta}$ are pairwise disjoint. We prove this fact now. Suppose there are $\theta \neq \theta^{\prime}$ with $J_{\theta} \cap J_{\theta^{\prime}} \neq \emptyset$. Find $k$ minimal such that $\theta_{k} \neq \theta_{k}^{\prime}$. Then clearly

$$
J_{\theta} \cap J_{\theta^{\prime}}=f^{\theta_{k}}\left(D_{k}\right) \cap f^{\theta_{k}^{\prime}}\left(D_{k}\right)=\{a\}
$$

for some single point $a \in X$. Taking the image by $f^{\alpha_{k}}$, we have

$$
f^{\alpha_{k}}(a) \in f^{\alpha_{k}}\left(J_{\theta}\right) \cap f^{\alpha_{k}}\left(J_{\theta^{\prime}}\right)=J_{\tau^{\alpha}(\theta)} \cap J_{\tau^{\alpha_{k}\left(\theta^{\prime}\right)}}=f^{\theta_{k}+\alpha_{k}}\left(D_{k}\right) \cap f^{\theta_{k}^{\prime}+\alpha_{k}}\left(D_{k}\right)=\{a\},
$$

because $D_{k}$ is periodic with period $\alpha_{k}$. This shows that $a$ is periodic with period $\alpha_{k}$. In particular, it does not belong to the infinite minimal set $M$. Now for $n \in \mathbb{N}$, let

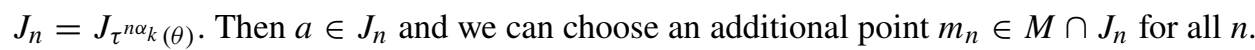
Thus the $J_{n}$ sets are non-degenerate subdendrites and intersect pairwise only at $a$. In particular, the sets $\left(a, m_{n}\right]$ are pairwise disjoint connected subsets of $X$, so their diameters must converge to zero (see e.g. [19, Lemma 2.3]). However, because $M$ is closed, this shows that $a \in M$, a contradiction.

Now that the sets $J_{\theta}, \theta \in \Omega$ have been shown to be pairwise disjoint, we see immediately that $\pi$ is well defined. It is also easy to see that $\pi$ is continuous and $\tau \circ \pi=\pi \circ f$.

Again, because the sets $J_{\theta}, \theta \in \Omega$ are pairwise disjoint connected sets in $X$, only countably many of them can have positive diameter. It follows that $\pi^{-1}(\theta)$ is a singleton except for countably many $\theta$. It remains to show that $M \cap J_{\theta}$ is countable when $J_{\theta}$ is non-degenerate. Because $M$ is minimal and $J_{\theta}$ never returns to itself, we must have $M \cap J_{\theta} \subset \operatorname{Bd}\left(J_{\theta}\right)$, where $\operatorname{Bd}\left(J_{\theta}\right)$ stands for the boundary of $J_{\theta}$. However, the boundary in $X$ of the subdendrite $J_{\theta}$ is a subset of $E\left(J_{\theta}\right) \cup B(X) \cup \overline{E(X)}$, which is countable by the assumption that $E(X)$ has countable closure. Here we use the well-known facts that $B(X)$ is countable in any dendrite, and the cardinality of the endpoint set of a dendrite cannot increase when we pass to a subdendrite, see e.g. [21, 22]. 
Remark 3.3. We have in fact shown that for a zero entropy map on a dendrite whose endpoint set has countable closure, every minimal subsystem is a regular extension of its maximal equicontinuous factor in the sense defined in [12]. For infinite minimal sets, this is implied by Lemma 3.2(3) and a finite minimal set is just a periodic orbit.

The next lemma strengthens [4, Lemma 3.5] by relaxing the condition that $E(X)$ be closed.

Lemma 3.4. [4, Lemma 3.5] Let $X, f, M,\left(D_{k}\right),\left(\alpha_{k}\right)$ be as in Proposition 3.1 and suppose that $\overline{E(X)}$ is countable. Then there is $N \geq 1$ such that for all $k \geq N, f^{i_{k}}\left(D_{k}\right)$ is a free arc for some $0 \leq i_{k} \leq \alpha_{k}-1$.

Proof. Using Lemma 3.2, we know that there are uncountably many singleton sets $J_{\theta}$. Now a dendrite whose endpoint set has countable closure is always the union of a countable sequence of free arcs and a countable set, see [5, Theorem 2.2]. It follows that we can find $\theta$ with the singleton $J_{\theta}$ in the interior of a free $\operatorname{arc} A$ in $X$. Because $J_{\theta}$ is the nested intersection $\bigcap_{N} f^{\theta_{N}}\left(D_{N}\right)$, we can find $N$ large enough that $f^{\theta_{N}}\left(D_{N}\right)$ is contained in $A$. Then $f^{\theta_{k}}\left(D_{k}\right)$ is a free arc for all $k \geq N$.

Our next result is a good first step in showing that recurrent points are minimal. It is a modified version of [3, Theorem 1.1], and the proof closely follows the one from that paper.

LeMmA 3.5. Let $X$ be a dendrite with $\overline{E(X)}$ countable, $f: X \rightarrow X$ a continuous map with zero topological entropy, and $x \in X$ a point which is recurrent but not periodic. Then $\omega_{f}(x)$ contains no periodic points.

Proof. Throughout the proof, we will use freely the following well-known property of $\omega$-limit sets (e.g. [6]): if for fixed $n \geq 2$ we write $W_{i}=\omega_{f^{n}}\left(f^{i}(x)\right)$ for $0 \leq i<n$, then $\omega_{f}(x)=\bigcup_{i=0}^{n-1} W_{i}$ and $f\left(W_{i}\right)=W_{i+1}(\bmod n)$. In particular, if $\omega_{f}(x)$ is uncountable, then so is each $W_{i}$, and if $\omega_{f}(x)$ contains a given fixed point, then each $W_{i}$ contains it as well. We continue to use the notation $[x, y]$ for the unique $\operatorname{arc}$ in $X$ with endpoints $x, y \in X$, and if $z \in(x, y)=[x, y] \backslash\{x, y\}$, we will say for simplicity that $z$ lies between $x$ and $y$.

Now let $L=\omega_{f}(x)$, where $x$ is recurrent but not periodic. Then $L$ is the closure of the orbit of $x$, and hence it is a perfect uncountable set.

Step 1: L does not contain a periodic point with a free arc neighborhood in X. Suppose to the contrary that $a \in L, f^{N}(a)=a$, and some free $\operatorname{arc} C$ is a neighborhood of $a$ in $X$. Then, by the standard properties mentioned above, $a \in \omega_{f^{N}}\left(f^{i}(x)\right)$ for some $0 \leq i<N$. Replacing $f$ with its iterate and $x$ with its image, we may safely assume that $N=1$ and $i=0$, that is, $a$ is a fixed point in $L=\omega_{f}(x)$.

Because periodic points are never isolated in infinite $\omega$-limit sets, we know that $L$ accumulates on $a$ from at least one side in the free $\operatorname{arc} C$. So, choose an endpoint $b$ of $C$ such that $L \cap[a, b]$ accumulates on $a$. For convenience, we let $C$ carry its natural order as an arc, oriented in such a way that $a<b$. Choose five points $y_{i} \in L \cap[a, b]$ 
with $a<y_{1}<y_{2}<y_{3}<y_{4}<y_{5}<b$. Choose three small arc neighborhoods $I_{2}, I_{3}, I_{4}$ containing $y_{2}, y_{3}, y_{4}$, respectively, and let them be pairwise disjoint and lie between $y_{1}$ and $y_{5}$. Because the orbit of $x$ visits each of these neighborhoods $I_{i}$ infinitely often, there must be points in $I_{2}$ and $I_{4}$ which visit $I_{3}$, so by [19, Theorem 2.13], $f$ has a periodic point $c$ between $y_{1}$ and $y_{5}$. Replacing $x$ with a point from its orbit near $y_{1}$, we may assume that $a<x<c<y_{5}<b$. Let $r$ be the period of $c$ and put $g=f^{r}$. Because $a$ was already fixed for $f$, we have $a \in \omega_{g}(x)$ as well. Note that because $x$ is recurrent for $f$, it is also recurrent for $g$.

CLAIM. There is an arc I invariant for $g$ with $[a, x] \subseteq I \subseteq[a, c]$.

Proof of Claim. To prove the claim, put $I=\overline{\bigcup_{n=0}^{\infty} g^{n}([a, x])}$. Because $a$ is fixed and $x$ is recurrent, it suffices to show that $g^{n}([a, x]) \subseteq[a, c]$ for all $n$. If this is not true, then there is $z \in[a, x]$ and $n_{0} \geq 1$ such that $a$ is between $g^{n_{0}}(z)$ and $c$ or $c$ is between $g^{n_{0}}(z)$ and $a$. We treat these two cases separately.

Suppose first that $a$ is between $g^{n_{0}}(z)$ and $c$. Then $a \in g^{n_{0}}([z, c])$, so there is $a_{-1}$ between $z$ and $c$ with $g^{n_{0}}\left(a_{-1}\right)=a$. Then $f^{n}\left(a_{-1}\right)=a$ for all $n \geq n_{0} \cdot r$. Because $L \cap[a, b]$ accumulates on $a$, we can find a point $x^{\prime} \in \operatorname{Orb}_{f}(x)$ between $a$ and $a_{-1}$. Because $y_{5} \in \omega_{f}(x)$, we can find $n \geq n_{0} \cdot r$ such that $f^{n}\left(x^{\prime}\right)$ is close to $y_{5}$ and $a<x^{\prime}<a_{-1}<c<$ $f^{n}\left(x^{\prime}\right)$. Put $J=\left[a, x^{\prime}\right]$ and $K=\left[x^{\prime}, a_{-1}\right]$. Then $f^{n}(J) \cap f^{n}(K) \supseteq J \cup K$, so $f$ possesses an arc horseshoe and thus has positive topological entropy, a contradiction.

Suppose instead that $c$ is between $g^{n_{0}}(z)$ and $a$. Then $c \in g^{n_{0}}([a, x])$, so there must be $c_{-1}$ between $a$ and $x$ with $g^{n_{0}}\left(c_{-1}\right)=c$. Because $a \in \omega_{g}(x)$, we can choose $n>n_{0}$ with $g^{n}(x)$ close to $a$ so that $g^{n}(x)<c_{-1}<x<c$. Put $J=\left[c_{-1}, x\right]$ and $K=[x, c]$. Then again $g^{n}(J) \cap g^{n}(K) \supseteq J \cup K$, so $g$ has positive topological entropy and so does $f$, a contradiction. This completes the proof of the claim.

Now we may use the claim to finish Step 1. Because $x$ belongs to the closed invariant set $I$, we have $\omega_{g}(x)=\omega_{\left.g\right|_{I}}(x)$. However, $\left.g\right|_{I}$ is an interval map, and when an infinite $\omega$-limit set for an interval map contains a periodic point, the topological entropy must be positive (see [20]), a contradiction.

Step 2: $L$ does not contain any periodic points. Suppose to the contrary that $a \in L$ is a periodic point. As in Step 1, we may assume that $a$ is fixed. By [5, Theorem 2.2], the dendrite $X$ is the union of a countable sequence of free arcs together with a countable set. In particular, we can find a free $\operatorname{arc} C$ not containing $a$ with $L \cap C$ uncountable. Write $C=$ $[u, v]$ with $v$ between $u$ and $a$ and let $<$ denote the order in $C$ with $u<v$. Because $L \cap C$ is infinite, we may choose four points $x_{i} \in \operatorname{Orb}_{f}(x)$ with $u<x_{1}<x_{2}<x_{3}<x_{4}<v$. As in Step 1, we can use small arc neighborhoods of $x_{2}, x_{3}, x_{4}$ to find a periodic point $c$ with $u<x_{1}<c<v$, and because $x_{1}$ is in the orbit of $x$, we may redefine $x=x_{1}$ without changing $\omega_{f}(x)$. Let $r$ denote the period of $c$ and put $g=f^{r}$. Because $x$ is recurrent also for $g$, we have $\operatorname{Orb}_{g}(x) \cap[u, c]$ infinite, so we can find two points $x_{5}, x_{6} \in \operatorname{Orb}_{g}(x)$ with $u<x_{5}<x_{6}<c$ and passing forward along the orbit, we can redefine $x=x_{6}$ without changing $\omega_{g}(x)$. In particular, $x_{5} \in \omega_{g}(x)=\overline{\operatorname{Orb}_{g}(x)}$, so we can choose $p \geq 1$ with $g^{p}(x)$ close to $x_{5}$ so that $u<g^{p}(x)<x<c$. 
Let $l=\omega_{g} p(x)$. We have $x \in l$ because $x$ is recurrent and $a \in l$ because $a$ is a fixed point in $\omega_{f}(x)$. Moreover, $c \notin l$ as a result of Step 1 . So let $X_{0}, X_{1}$ denote the connected components of $X \backslash\{c\}$ containing $x$ and $a$, respectively, and put $l_{i}=l \cap X_{i}$. Then $l=$ $l_{0} \cup l_{1}$ expresses $l$ as the disjoint union of two non-empty open subsets (in the topology induced from $X$ to $l$ ). Recall that every $\omega$-limit set $\omega_{f}(x)$ is weakly incompressible, that is, $f(\bar{U}) \not \subset U$ for any set $U \subsetneq \omega_{f}(x)$ open in $\omega_{f}(x)$ (see, e.g. [25]). Thus we have $g^{p}\left(l_{0}\right) \cap$ $l_{1} \neq \emptyset$. Therefore, we may choose $y \in l_{0}$ with $g^{p}(y) \in l_{1}$, and because $\operatorname{Orb}_{g} p(x)$ is dense in $l_{0}$, we may choose $y$ from the orbit of $x$. We finish the proof in two cases, depending on the location of $y$.

Suppose first that $y$ is between $x$ and $c$. In the ordering of the $\operatorname{arc}\left[g^{p}(x), g^{p}(y)\right]$, we have $g^{p}(x)<x<y<c<g^{p}(y)$. Put $I=[x, y]$ and $J=[y, c]$. Clearly $g^{p}(I) \supseteq I \cup J$. Because $y \in \operatorname{Orb}_{g}(x)$, we have $\omega_{g}(y)=\omega_{g}(x) \supset \operatorname{Orb}_{g}(x) \ni g^{p}(x)$. In particular, we may choose $n>p$ to make $g^{n}(y)$ as close to $g^{p}(x)$ as we like, so that $x, y \in\left[g^{n}(y), c\right]$. However, then $g^{n}(J) \supseteq I \cup J$. We conclude that $g$ possesses an arc horseshoe and thus $g$ has positive topological entropy, which is a contradiction with $h_{\text {top }}(f)=0$.

Suppose instead that $x$ is between $y$ and $c$. Then $c \in\left[g^{p}(x), g^{p}(y)\right]$, so there is $c_{-1} \in(x, y)$ with $g^{p}\left(c_{-1}\right)=c$. In the ordering of the arc $\left[y, g^{p}(y)\right]$, we have $y<$ $c_{-1}<x<c$ and we also have $x \in\left(g^{p}(x), c\right)$. Put $I=\left[c_{-1}, x\right]$ and $J=[x, c]$. Because $y \in \operatorname{Orb}_{g}(x) \subset \omega_{g}(x)$, we can find $n>p$ with $g^{n}(x)$ as close to $y$ as we like. In particular, we can get $x, c_{-1} \in\left[g^{n}(x), c\right]$. However then $g^{n}(I) \cap g^{n}(J) \supseteq I \cup J$. Again we conclude that $g$ has positive topological entropy, which is a contradiction. This ends the proof.

THEOREM 3.6. If $X$ is a dendrite in which $\overline{E(X)}$ is countable and if $f: X \rightarrow X$ has zero topological entropy, then every recurrent point for $f$ is minimal.

Proof. Let $x \in \operatorname{Rec}(f)$. If $x$ is periodic, then it is minimal, so assume $x$ is not periodic. Let $L=\omega(x)$. Let $M \subset L$ be a minimal set. By Lemma 3.5, $L$ contains no periodic orbits, so $M$ is an infinite minimal set. Then Proposition 3.1 applies and we get a sequence of $f$-periodic subdendrites $\left(D_{k}\right)_{k \geq 1}$ and periods $\left(\alpha_{k}\right)$ satisfying properties (1)-(5) of that proposition. By Lemma 3.4 for all sufficiently large $k$, we have that $f^{i}\left(D_{k}\right)$ is a free arc for suitable $i$. Because $M$ is infinite and $D_{k}$ is periodic, we have $M \cap$ int $f^{i}\left(D_{k}\right) \neq \emptyset$ and as a consequence, $\operatorname{Orb}_{f}(x) \cap D_{k} \neq \emptyset$, for every sufficiently large $k$. Hence, $\bigcap_{k \geq 1} \operatorname{Orb}_{f}\left(D_{k}\right)$ contains $L$, that is, property (4) still holds with $L$ in the place of $M$.

We claim that property (5) also holds with $L$ in the place of $M$. Fix $k$ and denote $L_{i}=$ $f^{i}\left(D_{k}\right) \cap L$ for $0 \leq i<\alpha_{k}-1$. Observe that $L$ does not contain periodic points, and the set $\operatorname{Orb}\left(f^{i}\left(D_{k}\right) \cap f^{j}\left(D_{k}\right)\right)$ is always finite and invariant for any $i \neq j$ (can be empty) and hence $L_{i} \cap f^{j}\left(D_{k}\right)=\emptyset$ for $i \neq j$. This shows that the sets $L_{i} \cap L_{j}=\emptyset$ for $i \neq j$. Clearly $f\left(L_{i}\right) \subseteq L_{i+1\left(\bmod \alpha_{k}\right)}$, and $f(L)=L$ because $\omega$-limit sets are always mapped onto themselves. This shows that $f\left(L_{i}\right)=L_{i+1\left(\bmod \alpha_{k}\right)}$. In particular, we conclude that $L_{i}$ is uncountable for each $i$.

Again using Lemma 3.4, choose $k$ large enough that $f^{i}\left(D_{k}\right)$ is a free arc for some $0 \leq$ $i<\alpha_{k}-1$ and let $A=f^{i}\left(D_{k}\right)$ denote that free arc. We have just shown that $L_{i}=A \cap L$ is uncountable, so because $A$ is a free arc, there are points from $\omega(x)$ in its interior. Thus we can find a point $y=f^{l}(x)$ from the forward orbit of $x$ in $A$. Then $\omega_{f}(x)=\omega_{f}(y)$ and 
$y$ is also recurrent for $f$. Because $\operatorname{Rec}\left(f^{\alpha_{k}}\right)=\operatorname{Rec}(f), y$ is also recurrent for $f^{\alpha_{k}}$. However, the restriction of $f^{\alpha_{k}}$ to $A$ is an interval map with zero topological entropy. For such a map, all recurrent points are minimal points, see e.g. [6, Ch. VI. Proposition 7]. Thus, $y$ is a minimal point for $f^{\alpha_{k}}$, and hence also for $f$. This shows that $\omega_{f}(y)=\omega_{f}(x)$ is a minimal set, and hence $x$ itself is minimal.

\section{Discrete spectrum in dendrites with $\overline{E(X)}$ countable}

By [17, Theorem 1.5], each one-sided subshift with zero entropy can be extended to a dynamical system on the Gehman dendrite with zero topological entropy. This provides a plethora of examples of dynamical systems on a dendrite with a closed set of endpoints having zero topological entropy and invariant measures which do not have discrete spectrum. However, in the Gehman dendrite, $E(X)$ is uncountable, because $E(X)$ is a Cantor set. Alternatively, each dendrite with $E(X)$ uncountable contains a copy of the Gehman dendrite (see e.g. [2], cf. [17]). So on all these dendrites, there exist dynamical systems with zero topological entropy and invariant measures not having discrete spectrum.

Our work below shows that the opposite holds in the case of a dendrite $X$, where $\overline{E(X)}$ is countable: all invariant measures of zero-entropy mappings have discrete spectrum. So in the case of dendrites, the remaining case in Question 1.1 is when $E(X)$ is countable but $\overline{E(X)}$ is uncountable. This case is left as a problem for further research.

LEMMA 4.1. Let $(X, f)$ be a topological dynamical system and suppose that all measures $\mu \in M_{f}(X)$ which are concentrated on $A_{i}$ have discrete spectrum, for each member $A_{i}$ of some finite or countable collection of invariant Borel sets. Then any $\mu \in M_{f}(X)$ which is concentrated on $\bigcup_{i} A_{i}$ also has discrete spectrum. In particular, if $\operatorname{Rec}(f) \subseteq \bigcup_{i} A_{i}$, then every $\mu \in M_{f}(X)$ has discrete spectrum.

Proof. Let $\mu$ be any finite invariant measure concentrated on $\bigcup_{i} A_{i}$. Because each $A_{i}$ is invariant, that is, $f\left(A_{i}\right) \subset A_{i}$, and $f$ preserves $\mu$, we may assume by throwing away a set in $X$ of $\mu$-measure zero that $f^{-1}\left(A_{i}\right)=A_{i}$ for each $i$.

We may take the index set for the variable $i$ to be $\{1, \ldots, n\}$ in the finite case or $\mathbb{N}$ in the countable case. Then putting $B_{i}=A_{i} \backslash \bigcup_{j<i} A_{j}$ for each $i$, we get a collection $\left\{B_{i}\right\}$ of pairwise disjoint invariant Borel sets. Now let $I=\left\{i: \mu\left(B_{i}\right)>0\right\}$ and write $\mu_{i}=\left.\mu\right|_{B_{i}}$ for the (unnormalized) restriction of $\mu$ to $B_{i}$. Then we get a direct sum decomposition of Hilbert spaces $L_{\mu}^{2}(X)=\bigoplus_{i \in I} L_{\mu_{i}}^{2}\left(B_{i}\right)$. (Here in a direct sum $\bigoplus H_{i}$ of Hilbert spaces, we include all $\left(v_{i}\right), v_{i} \in H_{i}$ such that $\sum\left\|v_{i}\right\|^{2}<\infty$. We do not require that all but finitely many $v_{i}$ vanish.) We may extend each function $\phi \in L_{\mu_{i}}^{2}\left(B_{i}\right)$ to an element of $L_{\mu_{i}}^{2}(X)$ by letting $\phi$ vanish outside of $B_{i}$. Because $f^{-1}\left(B_{i}\right)=B_{i}$, we see that if $\phi \circ f=\lambda \phi$ holds $\mu_{i}$-almost everywhere (a.e) in $B_{i}$, then by letting $\phi$ vanish outside $B_{i}$, it continues to hold $\mu$-a.e in $X$. Thus we have the equivalent direct sum decomposition

$$
L_{\mu}^{2}(X)=\bigoplus_{i \in I} L_{\mu_{i}}^{2}(X)
$$

and an eigenfunction in a coordinate space is still an eigenfunction in the whole space. For each $i \in I$, the normalized measure $\mu_{i} / \mu\left(B_{i}\right)$ is an invariant probability measure for $f$ concentrated on $B_{i} \subset A_{i}$, so by hypothesis, the eigenfunctions of the Koopman operator 
on the space $L_{\mu_{i} / \mu\left(B_{i}\right)}^{2}(X)$ have dense linear span. Dropping the normalizing constant, the same holds for $L_{\mu_{i}}^{2}(X)$. Passing through the direct sum decomposition, it follows that the eigenfunctions of the Koopman operator on the space $L_{\mu}^{2}(X)$ have dense linear span, that is, $\mu$ has discrete spectrum.

The last statement of the lemma follows by the Poincaré recurrence theorem, whereby if $\operatorname{Rec}(f) \subseteq \bigcup A_{i}$, then every measure $\mu \in M_{f}(X)$ is concentrated on $\bigcup A_{i}$.

LeMmA 4.2. Let $X$ be a dendrite and suppose that $f: X \rightarrow X$ is a continuous map with zero topological entropy. If $D \subset X$ is a tree and $R: X \rightarrow D$ is a natural retraction, then the map $F: D \rightarrow D$ given by $F=R \circ f$ has zero topological entropy.

Proof. Suppose that $F$ has positive entropy. Then by [15], there exists an arc horseshoe $I_{1}, I_{2}$ with $F^{n_{1}}\left(I_{1}\right) \cap F^{n_{2}}\left(I_{2}\right) \supset I_{1} \cup I_{2}$ for some $n_{1}, n_{2} \in \mathbb{N}$. Then $F^{i}\left(I_{j}\right)$ is not a single point for any $i=1, \ldots, n_{j}$ and $j=1,2$. However, if $F(J)$ is non-degenerate for an arc $J$, then $f(J) \supset F(J)$ which implies that $f^{n_{1}}\left(I_{1}\right) \cap f^{n_{2}}\left(I_{2}\right) \supset I_{1} \cup I_{2}$ which implies that $f$ has positive topological entropy. A contradiction.

THEOREM 4.3. Let $X$ be a dendrite such that $\overline{E(X)}$ is countable and let $f: X \rightarrow X$ be a continuous map with zero topological entropy. Then every measure $\mu \in M_{f}(X)$ has discrete spectrum.

Proof. Let $Z=\left\{z \in \overline{E(X)}: \omega_{f}(z)\right.$ is an infinite minimal set $\}$. Following arguments in [21, Theorem 10.27], let $\left(T_{n}\right)_{n \in \mathbb{N}} \subset X$ be an increasing sequence of topological trees with endpoints in $E(X)$ defined as follows. We inductively construct the sequence $\left(T_{n}\right)_{n \in \mathbb{N}}$ starting with $T_{1}=\left\{e_{1}\right\}$ for some $e_{1} \in E(X)$. Then for $n \geq 1$, we attach to $T_{n}$ an arc $\left[e, e_{n+1}\right]$ whose one endpoint $e_{n+1}$ belongs to $E(X) \backslash T_{n}$ and $e \in T_{n}$. Because $E(X)$ is countable, we can put every endpoint into one of the trees, that is, we let the sequence $\left(e_{n}\right)_{n \in \mathbb{N}}$ be an enumeration of $E(X)$, and then $\bigcup_{n \geq 1} T_{n}$ being a connected set must coincide with the whole dendrite $X$.

Let $\hat{T}_{n}=\bigcap_{i=0}^{\infty} f^{-i}\left(T_{n}\right)$ be the maximal invariant set completely contained in $T_{n}$. Let $\operatorname{Per}(f)$ be the set of periodic points of $f$. We claim that

$$
\operatorname{Rec}(f) \subset \operatorname{Per}(f) \cup\left(\bigcup_{z \in Z} \omega_{f}(z)\right) \cup\left(\bigcup_{n} \hat{T}_{n}\right) .
$$

To see this, let $x$ be a non-periodic recurrent point whose orbit is not contained in any of the trees $T_{n}$. This means that there are points $f^{n_{i}}(x)$ which belong to $T_{m_{i}} \backslash T_{m_{i}-1}$ for some strictly increasing sequences $m_{i}, n_{i} \rightarrow \infty$. Then the $\operatorname{arcs}\left[f^{n_{i}}(x), e_{m_{i}}\right]$ in $X$ are pairwise disjoint, so by [19, Lemma 2.3], their diameters tend to zero. This shows that $\lim _{\inf _{n \rightarrow \infty}} d\left(f^{n}(x), E(X)\right)=0$. Therefore, $\omega_{f}(x) \cap \overline{E(X)} \neq \emptyset$. By Theorem 3.6, $\omega_{f}(x)$ is a minimal set, so choosing $z \in \omega_{f}(x) \cap \overline{E(X)}$, we have $\omega_{f}(x)=\omega_{f}(z)$. This establishes equation (4.2).

Now observe that any finite invariant measure concentrated on $\operatorname{Per}(f)$ has discrete spectrum, see e.g. [17, Theorem 2.3]. As for the sets $\hat{T}_{n}$, note that for each $n \in \mathbb{N}$, the map $F=R \circ f$, where $R: X \rightarrow T_{n}$ is a retraction, satisfies $\left.F\right|_{\hat{T}_{n}}=\left.f\right|_{\hat{T}_{n}}$ by the definition 
and therefore each $f$-invariant measure concentrated on $\hat{T}_{n}$ (a subset of a tree) has discrete spectrum, as, by [17], all invariant measures of $F$ have discrete spectrum.

Finally, we claim that any invariant measure concentrated on $\omega_{f}(z), z \in Z$, has discrete spectrum. Let $\left(D_{k}\right)$ be the periodic subdendrites with periods $\left(\alpha_{k}\right)$ described in Proposition 3.1 and let $\pi:\left(\omega_{f}(z), f\right) \rightarrow(\Omega, \tau)$ be the factor map onto the odometer described in Lemma 3.2. Let $\mu \in M_{f}(X)$ be any invariant measure concentrated on $\omega_{f}(z)$. Then the pushforward measure $\pi_{*}(\mu)$ is invariant for the odometer, so by unique ergodicity, it is the Haar measure on $\Omega$ and it has discrete spectrum as a consequence of [26, Theorem 3.5]. Now because $\omega_{f}(z)$ contains no periodic points, we know that $\mu$ is non-atomic and therefore countable sets have measure zero. Then in the category of measure preserving transformations, the factor map $\pi:\left(\omega_{f}(z), \mu, f\right) \rightarrow\left(\Omega, \pi_{*}(\mu), \tau\right)$ is in fact an isomorphism, because by Lemma 3.2, it is invertible except on a set of $\mu$-measure zero. This implies that $\mu$ has discrete spectrum.

We have shown that an invariant measure concentrated on any of the countably many invariant sets in equation (4.2) has discrete spectrum. By Lemma 4.1, this completes the proof.

Acknowledgements. We would like to thank M. Gröger and F. García-Ramos for pointing out the fact mentioned in Remark 3.3. M. Foryś-Krawiec was supported in part by the National Science Centre, Poland (NCN), grant SONATA BIS no. 2019/34/E/ST1/00237: 'Topological and Dynamical Properties in Parameterized Families of Non-Hyperbolic Attractors: the inverse limit approach'. S. Roth was supported by Czech Republic RVO funding for IČ47813059.

This research is part of a project that has received funding from the European Union's Horizon 2020 research and innovation programme under the Marie Skłodowska-Curie grant agreement no. 883748.

\section{REFERENCES}

[1] R. L. Adler, A. G. Konheim and M. H. McAndrew. Topological entropy. Trans. Amer. Math. Soc. 114 (1965), 309-319.

[2] D. Arévalo, W. J. Charatonik, P. Pellicer Covarrubias and L. Simón. Dendrites with a closed set of end points. Topology Appl. 115(1) (2001), 1-17.

[3] G. Askri. Li-Yorke chaos for dendrite maps with zero topological entropy and $\omega$-limit sets. Discrete Contin. Dyn. Syst. 37(6) (2017), 2957-2976.

[4] G. Askri. Equicontinuity and Li-Yorke pairs of dendrite maps. Dyn. Syst. 35(4) (2020), 597-608.

[5] A. Bartoš, J. Bobok, P. Pyrih, S. Roth and B. Vejnar. Constant slope, entropy, and horseshoes for a map on a tame graph. Ergod. Th. \& Dynam. Sys. 40(11) (2020), 2970-2994.

[6] L. S. Block and W. A. Coppel. Dynamics in One Dimension (Lecture Notes in Mathematics, 1513). Springer-Verlag, Berlin, 1992.

[7] R. Bowen. Entropy for group endomorphisms and homogeneous spaces. Trans. Amer. Math. Soc. 153 (1971), 401-414.

[8] H. Davenport. On some infinite series involving arithmetical functions (II). Q. J. Math. 8 (1937), 313-320.

[9] E. I. Dinaburg. The relation between topological entropy and metric entropy. Dokl. Akad. Nauk 190 (1970), $19-22$.

[10] T. Downarowicz. Entropy in Dynamical Systems (New Mathematical Monographs, 18). Cambridge University Press, Cambridge, 2011. 
[11] E. H. El Abdalaoui, G. Askri and H. Marzougui. Mobius disjointness conjecture for local dendrite maps. Nonlinearity 32(1) (2019), 285-300.

[12] F. García-Ramos, T. Jäger and X. Ye. Mean equicontinuity, almost automorphy and regularity. Israel J. Math. 243 (2021), 155-183.

[13] W. Huang, Z. Wang and X. Ye. Measure complexity and Möbius disjointness. Adv. Math. 347 (2019), 827-858.

[14] D. Karagulyan. On Möbius orthogonality for interval maps of zero entropy and orientation-preserving circle homeomorphisms. Ark. Mat. 53 (2015), 317-327.

[15] Z. Kočan, V. Kornecká-Kurková and M. Málek. Entropy, horseshoes and homoclinic trajectories on trees, graphs and dendrites. Ergod. Th. \& Dynam. Sys. 31 (2011), 165-175.

[16] J. Li, P. Oprocha, Y. Yang and T. Zeng. On dynamics of graph maps with zero topological entropy. Nonlinearity 30(12) (2017), 4260-4276.

[17] J. Li, P. Oprocha and G. H. Zhang. Quasi-graphs, zero entropy and measures with discrete spectrum. Preprint, 2021, arXiv:1809.05617v2 [math.DS].

[18] J. Li, S. Tu and X. Ye. Mean equicontinuity and mean sensitivity. Ergod. Th. \& Dynam. Sys. 35 (2015), 2587-2612.

[19] J. H. Mai and E. H. Shi. $\bar{R}=\bar{P}$ for maps of dendrites with Card(End (X)) < c. Internat. J. Bifur. Chaos Appl. Sci. Engrg. 19(4) (2009), 1391-1396.

[20] M. Misiurewicz. Horseshoes for continuous mappings of an interval. Dynamical Systems (Bresanone, 1978). Ed. C. Marchioro. Liguori, Naples, 1980, pp. 125-135.

[21] S. B. Nadler Jr. Continuum Theory. Marcel Dekker, New York, 1992.

[22] I. Naghmouchi. Pointwise-recurrent dendrite maps. Ergod. Th. \& Dynam. Sys. 33 (2013), 1115-1123.

[23] P. Sarnak. Three Lectures on the Möbius Function, Randomness and Dynamics (Lecture Notes). IAS, 2009.

[24] B. Scarpellini. Stability properties of flows with pure point spectrum. J. Lond. Math. Soc. (2) 26(3) (1982), 451-464.

[25] A. N. Sharkovsky, S. F. Kolyada, A. G. Sivak and V. V. Fedorenko. Dynamics of One-Dimensional Maps (Mathematics and Its Applications, 407). Kluwer Academic Publishers, Dordrecht, 1997.

[26] P. Walters. An Introduction to Ergodic Theory (Graduate Texts in Mathematics, 79). Springer, New York, 1982. 